\title{
CTRP3 attenuates cardiac dysfunction, inflammation, oxidative stress and cell death in diabetic cardiomyopathy in rats
}

\author{
Zhen-Guo Ma ${ }^{1,2,3} \cdot$ Yu-Pei Yuan ${ }^{1,2,3} \cdot$ Si-Chi Xu ${ }^{1,2,3} \cdot$ Wen-Ying Wei ${ }^{1,2,3}$. \\ Chun-Ru Xu ${ }^{1,2,3} \cdot$ Xin Zhang ${ }^{1,2,3} \cdot$ Qing-Qing Wu ${ }^{1,2,3} \cdot$ Hai-Han Liao ${ }^{1,2,3} \cdot$ Jian Ni ${ }^{1,2,3}$. \\ Qi-Zhu Tang $1,2,3,4$
}

Received: 4 December 2016 / Accepted: 3 February 2017 / Published online: 3 March 2017

(C) Springer-Verlag Berlin Heidelberg 2017

\begin{abstract}
Aims/hypothesis Oxidative stress, inflammation and cell death are closely involved in the development of diabetic cardiomyopathy (DCM). C1q/tumour necrosis factor-related protein-3 (CTRP3) has anti-inflammatory properties but its role in DCM remains largely unknown. The aims of this study were to determine whether CTRP3 could attenuate DCM and to clarify the underlying mechanisms.

Methods Streptozotocin (STZ) was injected intraperitoneally to induce diabetes in Sprague-Dawley rats. Cardiomyocytespecific CTRP3 overexpression was achieved using an adenoassociated virus system 12 weeks after STZ injection.

Results CTRP3 expression was significantly decreased in diabetic rat hearts. Knockdown of CTRP3 in cardiomyocytes at baseline resulted in increased oxidative injury, inflammation and apoptosis in vitro. Cardiomyocyte-specific overexpression of CTRP3 decreased oxidative stress and
\end{abstract}

Electronic supplementary material The online version of this article (doi:10.1007/s00125-017-4232-4) contains peer-reviewed but unedited supplementary material, which is available to authorised users.

Qi-Zhu Tang

qztang@whu.edu.cn

1 Department of Cardiology, Renmin Hospital of Wuhan University, Wuhan University, Jiefang Road 238, Wuhan 430060, People's Republic of China

2 Cardiovascular Research Institute of Wuhan University, Wuhan, People's Republic of China

3 Hubei Key Laboratory of Cardiology, Wuhan, People's Republic of China

4 Department of Cardiology, Renmin Hospital of Wuhan University, Cardiovascular Research Institute, Hubei Key Laboratory of Cardiology, Wuhan University, Jiefang Road 238, Wuhan 430060 , People's Republic of China inflammation, attenuated myocyte death and improved cardiac function in rats treated with STZ. CTRP3 significantly activated AMPactivated protein kinase $\alpha(\mathrm{AMPK} \alpha)$ and Akt (protein kinase B) in $\mathrm{H} 9 \mathrm{c} 2$ cells. CTRP3 protected against high-glucose-induced oxidative stress, inflammation and apoptosis in vitro. AMPK $\alpha$ deficiency abolished the protective effects of CTRP 3 in vitro and in vivo. Furthermore, we found that CTRP3 activated AMPK $\alpha$ via the $\mathrm{CAMP}-$ exchange protein directly activated by cAMP (EPAC)-mitogen-activated protein kinase kinase (MEK) pathway.

Conclusions/interpretation CTRP3 protected against DCM via activation of the AMPK $\alpha$ pathway. CTRP3 has therapeutic potential for the treatment of DCM.

Keywords AMPactivated protein kinase $\alpha \cdot$ Apoptosis $\cdot \mathrm{C} 1 \mathrm{q} /$ tumour necrosis factor-related protein-3 $\cdot \mathrm{CTRP} 3 \cdot$ Diabetic cardiomyopathy $\cdot$ Inflammation $\cdot$ Oxidative stress

$\begin{array}{ll}\text { Abbreviations } \\ \text { 4-HNE } & \text { 4-Hydroxynenal } \\ \text { AAV } & \text { Adeno-associated virus } \\ \text { AMPK } \alpha & \text { AMPactivated protein kinase } \alpha \\ \text { BAX } & \text { BCL-2-associated X protein } \\ \text { BCL-2 } & \text { B-cell lymphoma 2 } \\ \text { CaMKK } & \text { Calcium/calmodulin-dependent protein kinase } \\ & \text { kinase } \\ \text { CTRP } & \text { C1q/tumour necrosis factor-related protein } \\ \text { DCM } & \text { Diabetic cardiomyopathy } \\ \text { EPAC } & \text { Exchange protein directly activated by cAMP } \\ \text { FS } & \text { Fractional shortening } \\ \text { GFP } & \text { Green fluorescent protein } \\ \text { HG } & \text { High glucose } \\ \text { LKB1 } & \text { Liver kinase B1 } \\ \text { LPS } & \text { Lipopolysaccharide }\end{array}$




$\begin{array}{ll}\text { LVIDd } & \text { Left ventricular internal diastolic diameter } \\ \text { MEK } & \text { Mitogen-activated protein kinase kinase } \\ \text { MOI } & \text { Multiplicity of infection } \\ \text { PKA } & \text { Protein kinase A } \\ \text { PKC } & \text { Protein kinase C } \\ \text { PPAR- } \alpha & \text { Peroxisome proliferator-activated receptor- } \alpha \\ \text { ROS } & \text { Reactive oxygen species } \\ \text { ShRNA } & \text { Small hairpin RNA } \\ \text { SOD } & \text { Superoxide dismutase } \\ \text { STZ } & \text { Streptozotocin }\end{array}$

\section{Introduction}

Diabetic cardiomyopathy (DCM) is defined as ventricular dysfunction in diabetic individuals that cannot be ascribed to coronary artery disease and hypertension [1]. DCM is characterised by the loss of myocytes, myofibril depletion and accumulation of collagen [2] and has been identified as a major predisposing factor for heart failure in diabetic patients [3, 4]. The Framingham study reported that the heart failure rate in men and women with diabetes is, respectively, twice and five times that in people without diabetes [5]. The prevalence of diastolic dysfunction is as high as 40-60\% in diabetic individuals without coronary artery disease $[1,6]$. Moreover, the pathological changes of cardiomyopathy in patients with diabetes could not be effectively reversed after intensive blood glucose control [7]. Therefore, molecules that selectively inhibit the progression of DCM would be of great therapeutic interest.

Diabetic cardiac dysfunction is precisely coordinated by an orchestrated network comprised of numerous pathogenic factors. Emerging data have identified a key role for increased oxidative stress, inflammation and subsequent cell death [8-10]. Hyperglycaemia and inflammation all result in the overproduction of reactive oxygen species (ROS) [11], which induce lipid peroxidation and decrease antioxidant capacity, eventually leading to the loss of myocytes and cardiac dysfunction [12]. Additionally, ROS and myocyte death promote the transition from fibroblast to myofibroblast and interstitial fibrosis [13]. Therefore, developing an effective strategy to suppress the overproduction of ROS, inflammation and cell death in the heart is urgently needed for diabetic patients.

$\mathrm{C} 1 \mathrm{q} /$ tumour necrosis factor-related proteins (CTRPs), identified based on sequence homology with the globular domain of adiponectin, are recognised as novel secreted metabolic regulators [14]. CTRP3, expressed predominantly by adipose tissue, is also found in the heart and liver [15]. CTRP3 regulates hepatic glucose output [16], attenuates hepatic steatosis [17] and inhibits inflammatory responses [18, 19]. Although it has been shown to suppress apoptosis and reduce interstitial fibrosis in ischaemic mouse hearts $[15,20]$, CTRP3 has not been further investigated for additional biological activity in the heart, especially in cardiomyocytes.
Therefore, the aims of this study were to determine whether CTRP3 could protect against streptozotocin (STZ)-induced cardiac injury and to elucidate the potential mechanisms. To the best of our knowledge, this is the first report of a protective role for CTRP3 in DCM.

\section{Methods}

Materials See electronic supplementary material (ESM) Methods for full details.

Animals and animal model All animal experimental procedures followed National Institutes of Health guidelines and the guidelines of our hospital. Male Sprague-Dawley rats (8-9 weeks old, 180-220 g) were obtained from the Institute of Laboratory Animal Science, Chinese Academy of Medical Sciences (Beijing, China). The rats were allowed free access to food and water and were housed at a controlled temperature $\left(20-25^{\circ} \mathrm{C}\right)$ and humidity $(50 \pm 5 \%)$ on a $12 \mathrm{~h}$ light-dark cycle. After being fasted for $12 \mathrm{~h}$, the rats were given a single intraperitoneal injection of STZ $(65 \mathrm{mg} / \mathrm{kg})$. Rats with fasting blood glucose $>13.9 \mathrm{mmol} / \mathrm{l}$ in three independent measurements were defined as having diabetes and were used for further studies. After 12 weeks of diabetes, the rats were given a single tail-vein injection of adeno-associated virus (AAV)Ctrp3 (also known as C1qtnf3) or AAV-green fluorescent protein $(G f p)$ at a dose of $5 \times 10^{11}$ particles per rat. The rats were also infused subcutaneously with the recombinant human globular domain of CTRP3 $\left(0.2 \mu \mathrm{g} \mathrm{g}^{-1}\right.$ day $\left.^{-1}\right)$ via an Alzet osmotic minipump (Durect, Cupertino, CA, USA) for 2 weeks beginning 12 weeks post STZ injection. Diabetic rats were grouped by a random number table. The treatment and subsequent analyses were performed in a blind fashion for all groups. See ESM Methods for further details.

Echocardiographic and haemodynamic measurements Echocardiography was performed as previously described [21-23]. Haemodynamic variables were analysed using a microtip transducer catheter (SPR-839; Millar Instruments, Houston, TX, USA) and a Millar Pressure-Volume System (MPVS-400; Millar Instruments).

Histological analysis, immunofluorescence, immunohistochemistry and TUNEL staining The haematoxylin-eosin and Picrosirius Red staining were performed using standard procedures [21-23]. To detect the protein level of CTRP3 in hearts, sections were incubated with rabbit anti-rat CTRP3 antibody (1:100) and were observed under an Olympus DX51 fluorescence microscope (Olympus, Tokyo, Japan). For immunohistochemical analysis, sections were incubated with anti-4-hydroxynonenal (4-HNE, 1:100) or anti-TNF- $\alpha$ (1:100). TUNEL staining was performed using a commercial 
kit (Millipore, Billerica, MA, USA), following the manufacturer's instructions. See ESM Methods for details.

Adenoviral vector construction The source of adenoviral (Ad)-shAmpk $\alpha 2$ adenoviruses was described in our previous articles [21, 22]. Knockdown of CTRP3 was carried out using adenoviral vectors carrying Ctrp 3 small hairpin RNAs (shRNAs), which were generated by Hanbio (Shanghai, China). Scrambled shRNA was used as the control.

Cell culture and treatment Neonatal rat cardiomyocytes were isolated as previously described [24]. To detect expression of CTRP 3 , the myocytes were stained with anti- $\alpha$-actinin (1:100) and anti-CTRP3 (1:100). H9c2 cells were purchased from the Cell Bank of the Chinese Academy of Sciences (Shanghai, China), where mycoplasma detection, DNAfingerprinting and isozyme analyses were performed. Mycoplasma contamination was not detected in H9c2 cells. To investigate the protection of CTRP3, H9c2 cells were pretreated with recombinant human globular domain of CTRP3 $(3 \mu \mathrm{g} / \mathrm{ml})$ for $30 \mathrm{~min}$ followed by normal glucose $(5.5 \mathrm{mmol} / \mathrm{l})$ or high glucose (HG, $33 \mathrm{mmol} / \mathrm{l})$ treatment. To knock down AMP-activated protein kinase $\alpha 2$ (AMPK $\alpha 2), \mathrm{H} 9 \mathrm{c} 2$ cells were infected with Ad-shAmpko2 (multiplicity of infection $[\mathrm{MOI}]=150$ ) or Ad-shRNA for $4 \mathrm{~h}[21,22]$. To knock down CTRP3, H9c2 cells were infected with Ad-shCtrp3 $(\mathrm{MOI}=150)$ for $4 \mathrm{~h}$. For inhibition of Akt, H9c2 cells were incubated with Akt1/2 kinase inhibitor $(1 \mu \mathrm{mol} / \mathrm{l})$. To explore the mechanism by which CTRP3 activated AMPK $\alpha, \mathrm{H} 9 \mathrm{c} 2$ cells were incubated with H89 (a protein kinase A [PKA] inhibitor, $10 \mu \mathrm{mol} / \mathrm{l}$ ), 2'5'-ddAdo (an adenylate cyclase inhibitor, $200 \mu \mathrm{mol} / \mathrm{l}$ ), U0126 (a mitogen-activated protein kinase [MEK] inhibitor, $10 \mu \mathrm{mol} / \mathrm{l}$ ) or STO-609 (a calcium/ calmodulin-dependent protein kinase kinase $[\mathrm{CaMKK}]$ inhibitor, $800 \mathrm{nmol} / \mathrm{l})$. To knock down exchange protein directly activated by cAMP (EPAC), small interfering RNA (siEpac) was used. To induce cell death, $\mathrm{H} 9 \mathrm{c} 2$ cells were treated with TNF- $\alpha(5 \mathrm{ng} / \mathrm{ml}$ for $24 \mathrm{~h})$ or $\mathrm{H}_{2} \mathrm{O}_{2}(200 \mu \mathrm{mol}$ for $12 \mathrm{~h})$. See ESM Methods for further details.

\section{Western blot and reverse-transcription and real-time PCR} Western blot and real-time PCR were performed as previously described [21-23]. See ESM Methods and ESM Tables 1, 2 for information about the source, characteristics and validation of the antibodies.

Detection of total superoxide dismutase activity, NADPH oxidase activity, lipid peroxidation and cAMP The fresh rat hearts $(80-120 \mathrm{mg})$ were homogenised and then centrifuged (4230 $\mathrm{g}, 10 \mathrm{~min}$ ) to collect the supernatant fractions. The activity of total superoxide dismutase (SOD) and NADPH oxidase, as well as the level of lipid peroxidation, was measured by the commercial kits. The H9c2 cells were lysed to detect the levels of cAMP after CTRP3 treatment. cAMP was detected using a commercial kit.

Knockdown of AMPK $\alpha 2$ in the diabetic hearts To knock down AMPK $\alpha 2$ in the diabetic hearts, an intramyocardial injection of Ad-shAmpko2 was administered, as described previously for AAV-Ctrp3 and AAV-Gfp [21]. The level of Ampk $\alpha$ (also known as Prkaa2) mRNA was examined at different time points post infection. After 6 weeks, Doppler analysis and haemodynamic measurements were performed and the rats were euthanised.

Detection of ROS and cell viability H9c2 cells were cultured in six-well plates and were exposed to HG and CTRP3 for $24 \mathrm{~h}$. To detect ROS, the cells were incubated with $2^{\prime}, 7^{\prime}$ dichlorodihydrofluorescein diacetate (DCFH-DA) for $30 \mathrm{~min}$ at $37^{\circ} \mathrm{C}$. The production of ROS by the cells was observed under an Olympus IX53 fluorescence microscope. To quantify the production of ROS, the fluorescence intensity was measured using flow cytometry. Cell viability was determined using the cell counting assay (CCK-8; Dojindo Molecular Technologies, Rockville, MD, USA) following the manufacturer's protocol.

Statistical analyses The results in our study are presented as the mean \pm SEM. When comparing multiple groups, one-way ANOVA was used, followed by a post hoc Tukey's test. An unpaired, two-sided Student's $t$ test was used to compare differences between two groups. A repeated-measures ANOVA was also used. Sample size was determined as described in our previous study [21]. No samples have been excluded from analysis. Statistical significance was assigned at $p<0.05$.

\section{Results}

CTRP3 was downregulated in the diabetic hearts in vivo CTRP3 expression was assessed in cardiomyocytes isolated from neonatal rats. We found that CTRP 3 expression in cardiomyocytes was not as low as previously described (Fig. 1a) [25]. The level of CTRP3 protein in the heart was about 4.7 times higher than that in plasma and approximately half that in adipose tissue (Fig. 1b). Immunofluorescence analyses revealed that CTRP3 protein levels were dramatically reduced in hearts of diabetic rats (Fig. 1c). This was confirmed by western blot (Fig. 1d). These results indicate a role for CTRP3 in DCM.

CTRP3 deletion increased inflammation, oxidative stress and myocyte death at baseline Next, we investigated whether the reduced CTRP3 level affected cardiomyocytes. We knocked down CTRP3 in H9c2 cells. Downregulation of CTRP3 increased the mRNA levels of Tnf- $\alpha$ (also known as 
a
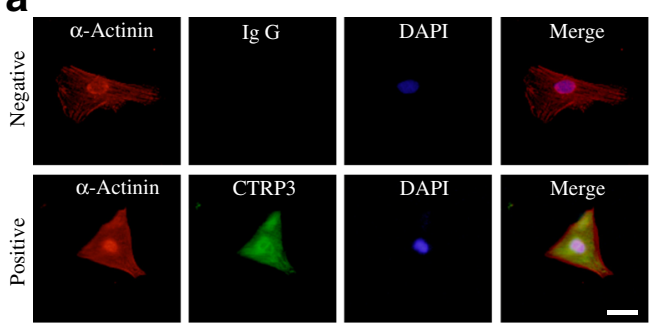

b

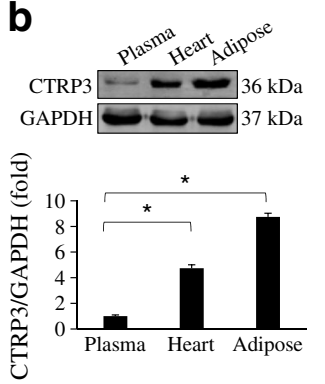

C
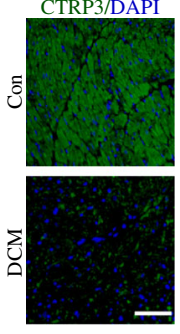

d

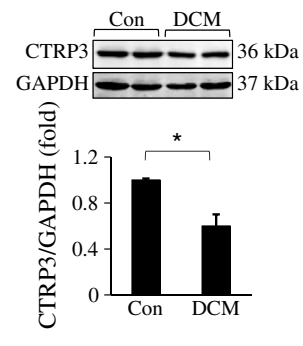

Fig. 1 CTRP3 expression was decreased in the diabetic rat hearts. (a) Representative images of immunofluorescence of CTRP3 in cardiomyocytes from neonatal rats $(n=6)$. (b) Protein level of CTRP3 $(n=6)$. (c) Immunofluorescence of CTRP3 in the diabetic hearts $(n=5)$. (d) Representative western blot analysis of CTRP3 in the hearts $(n=6)$. Scale bar, $100 \mu \mathrm{m}$. $* p<0.05$ for indicated comparisons. Con, control (no diabetes)

Tnf) and Mcp-1 (Ccl2) at baseline without affecting Il-6 (IlO) (Fig. 2a-d). CTRP3 deficiency also increased the mRNA level of P67phox (Ncf2) and ROS production in basal conditions without affecting P22phox (Cyba) (Fig. 2e-g). Moreover, knockdown of CTRP3 decreased the viability of H9c2 cells (Fig. 2h).

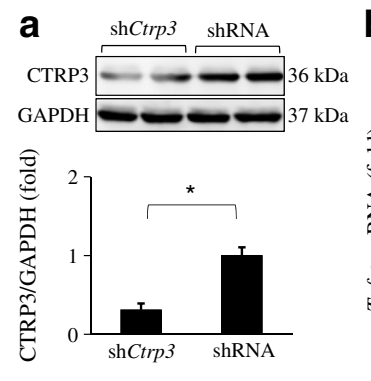

b

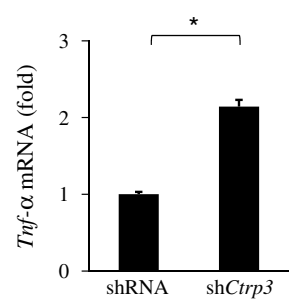

e

f
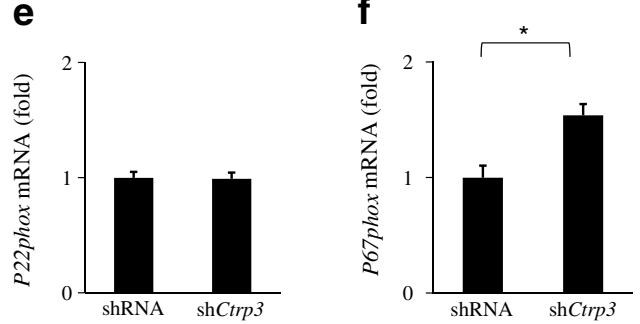

Fig. 2 CTRP3 deficiency increased inflammation, oxidative stress and cell death at baseline. (a) Protein level of CTRP3 in cardiomyocytes from neonatal rats $(n=6)$. (b-d) mRNA level of inflammatory factors after knockdown of CTRP3 in neonatal rat cardiomyocytes $(n=6)$. $(\mathbf{e}, \mathbf{f})$ mRNA level of P22phox (e) and P67phox (f) in neonatal rat
CTRP3 attenuated STZ-induced cardiac injury and improved cardiac function Rats treated with STZ exhibited typical symptoms of diabetes, including polydipsia and polyuria, along with increased blood glucose (ESM Table 3). STZ injection reduced body weight (ESM Table 4). Overexpression of CTRP3 in the hearts (Fig. 3a) did not affect blood glucose and body weight (ESM Table 3, 4). Rats treated with STZ injection for 12 weeks developed a decrement in cardiac function with a marked decrease in fractional shortening (FS) and left ventricular internal diastolic diameter (LVIDd); overexpression of CTRP3 in the hearts improved the decreased cardiac function (Fig. 3b, c). Compared with control rats (no overexpression of CTRP3), the rats treated with STZ displayed systolic dysfunction (dP/dt max 27\%) and diastolic dysfunction ( $\mathrm{dP} / \mathrm{dt} \min 25 \%$ ) (Fig. 3d, e). However, these diabetes-related cardiac functional alterations were attenuated after CTRP3 overexpression. Rats with diabetes had a decreased heart weight-to-body weight ratio and increased cell area and overexpression of CTRP3 protected against these pathological changes (Fig. 3f, g).

Next, we determined whether infusion of exogenous recombinant human CTRP3 would attenuate diabetesrelated cardiac dysfunction. Administration of CTRP3 slightly decreased blood glucose and significantly increased $\mathrm{dP} / \mathrm{dt} \max$ and $\mathrm{dP} / \mathrm{dt} \mathrm{min}$, indicating the therapeutic potential of CTRP3 in DCM (ESM Fig. 1a-c). Cardiac fibrosis in the diabetic rats was also evaluated. The collagen volume was increased in the diabetic hearts and was not affected by CTRP3 (ESM Fig. 2a, b). This was further confirmed by examining the mRNA levels of fibrotic markers (ESM Fig. 2c, d). c

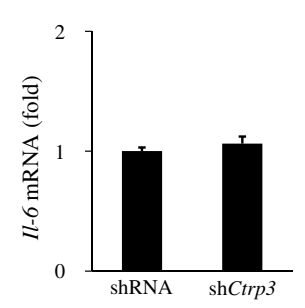

g

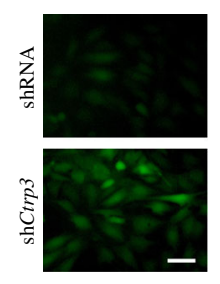

d

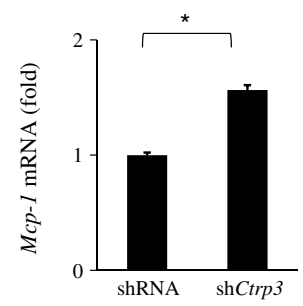

h

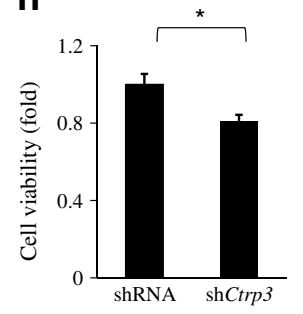

cardiomyocytes. (g) Representative fluorescence microscopy images of DCF-DA fluorescence in neonatal rat cardiomyocytes. Scale bar, $200 \mu \mathrm{m}$. (h) Cell viability after knockdown of CTRP3 in neonatal rat cardiomyocytes $(n=6)$. ' $p<0.05$ for indicated comparisons. 'shRNA' refers to scrambled control. 

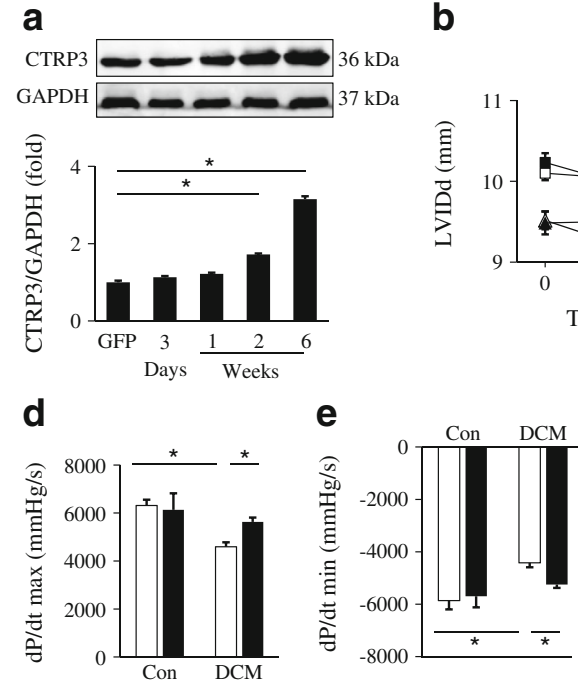

Fig. 3 CTRP3 improved diabetes-induced cardiac dysfunction. (a) Western blot analysis of CTRP3 in rat hearts after infection with an AAV $(n=5)$. (b, c) Alteration of LVIDd (b) and FS (c) after overexpression of CTRP3 $(n=6)$. White squares, control+GFP; black squares, control+CTRP3; white triangles, DCM+GFP; black triangles, DCM+

\section{Overexpression of CTRP3 in the diabetic rat hearts re-} duced oxidative damage As oxidative stress is a key feature of DCM, the effect of CTRP3 on oxidative injury was evaluated. Western blotting showed that overexpression of CTRP3 significantly decreased the NADPH oxidase subunit 67 phox and increased SOD2 expression in diabetic rat hearts (Fig. 4a). Our results also confirmed that the upregulation of $P 67 p h o x$ and Gp91phox (also known as $C y b b$ ) mRNA induced by STZ injection was reduced in diabetic rats with CTRP3 overexpression (Fig. 4b, c). CTRP3 overexpression in the diabetic rats also reduced the abnormal activity of NADPH oxidase (Fig. 4d). Compared with rats in the control groups, total SOD activity in the heart was significantly reduced in diabetic rats and was significantly increased by overexpression of CTRP3 in the hearts (Fig. 4e). CTRP3 overexpression also reduced the production of myocardial lipid peroxidation in the rats with diabetes (Fig. 4f). Immunohistochemical analyses of 4-HNE in the hearts also confirmed the attenuation of lipid peroxidation in the DCM+CTRP3 group compared with the DCM+GFP group (Fig. 4g).

CTRP3 prevented diabetes-induced inflammation in the hearts A previous study found that lower circulating levels of TNF- $\alpha$ were observed in CTRP3 transgenic mice [18]. In the present study, CTRP3 also had significant effects on myocardial Tnf- $\alpha$ and $M c p-1$ in rats with diabetes (Fig. 5a-d). However, CTRP3 had no effect on $I l-1 \beta$ (also known as $I l 1 b$ ) and $I l-6$ mRNA in diabetic rat hearts (Fig. 5a-d). Immunohistochemistry and western blot analysis also confirmed that TNF- $\alpha$ levels were significantly increased in the DCM+ GFP group but not in the DCM+CTRP3 group (Fig. 5e, f).
C

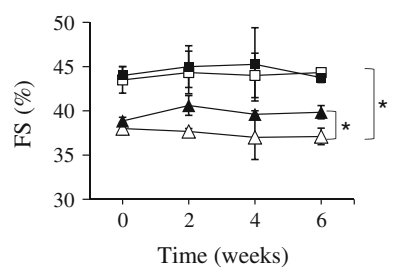

f

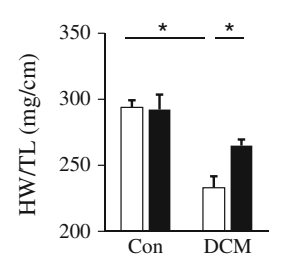

g

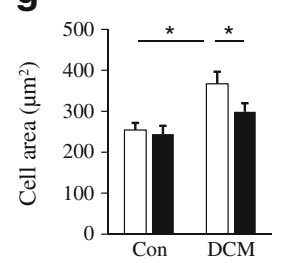

CTRP3. (d, e) Effect of CTRP3 on haemodynamic measurements $(n=8)$. (f) The ratio of heart weight $(\mathrm{HW})$ to tibia length (TL) $(n=10)$. (g) Sectional area of myocytes $(n=6)$. White bars, GFP; black bars, CTRP3. ${ }^{*} p<0.05$ for indicated comparisons. Con, control (no diabetes)

CTRP3 decreased diabetes-induced cell death in the hearts As shown in Fig. 5g, an increased proportion of apoptotic cells was observed in diabetic hearts and this was reduced significantly following CTRP3 overexpression. The inhibitory effects of CTRP3 on apoptosis were further confirmed by western blot results showing that CTRP3 upregulated the expression of B-cell lymphoma 2 (BCL-2) and reduced the expression of BAX (Fig. $5 g$, h).

CTRP3 activated AMPK $\alpha$ and Akt in the diabetic hearts Next, we investigated the precise mechanisms by which CTRP3 protected against diabetes-related cardiac injury. Compared with the control groups, rats with diabetes displayed decreased phosphorylation of Akt and AMPK in the hearts, and CTRP3 significantly increased the phosphorylation of AMPK $\alpha$ and Akt (Fig. 6). We also examined the phosphorylation of acetyl-CoA carboxylase, which reflects the activity of AMPK $\alpha$. As expected, CTRP3 promoted AMPK $\alpha$ activity (Fig. 6) in diabetic hearts. CTRP3 did not affect protein kinase $\mathrm{C}$ (PKC), nuclear factor-E2 p45-related factor 2 or peroxisome proliferator-activated receptor- $\alpha$ $($ PPAR $-\alpha)$ in diabetic hearts although it increased the level of PPAR- $\alpha$ in normal hearts (Fig. 6).

CTRP3 activated AMPK $\alpha$ and inhibited HG-induced ROS generation and inflammation in $\mathrm{H} 9 \mathrm{c} 2$ cells Given the observation that CTRP3 activated AMPK $\alpha$ and Akt in vivo, we examined the effects of CTRP3 on AMPK $\alpha$ and Akt in vitro and found that recombinant CTRP3 $(3 \mu \mathrm{g} / \mathrm{ml})$ significantly increased the phosphorylation of AMPK $\alpha$ and Akt (Fig. 7a). CTRP3 also increased the activity of acetyl- 
Fig. 4 CTRP3 attenuated diabetes-induced oxidative injury. (a) Western blot analysis of p67phox and SOD2 in diabetic rat hearts $(n=6)$. (b, c) PCR analysis of P67phox (b) and Gp91phox (c) in diabetic hearts $(n=6)$. (d) NADPH oxidase activity in diabetic hearts by CTRP3 overexpression $(n=7)$. (e) Total SOD activity in diabetic hearts after CTRP3 overexpression $(n=7)$. (f) Lipid peroxidation in diabetic hearts $(n=7)$. (g) Immunohistochemical analysis of 4-HNE in diabetic hearts. Representative images and quantification $(n=6)$ are shown. Scale bar, $300 \mu \mathrm{m}$. White bars, GFP; black bars, CTRP3. $* p<0.05$ for indicated comparisons. Con, control (no diabetes). MDA, malondialdehyde a
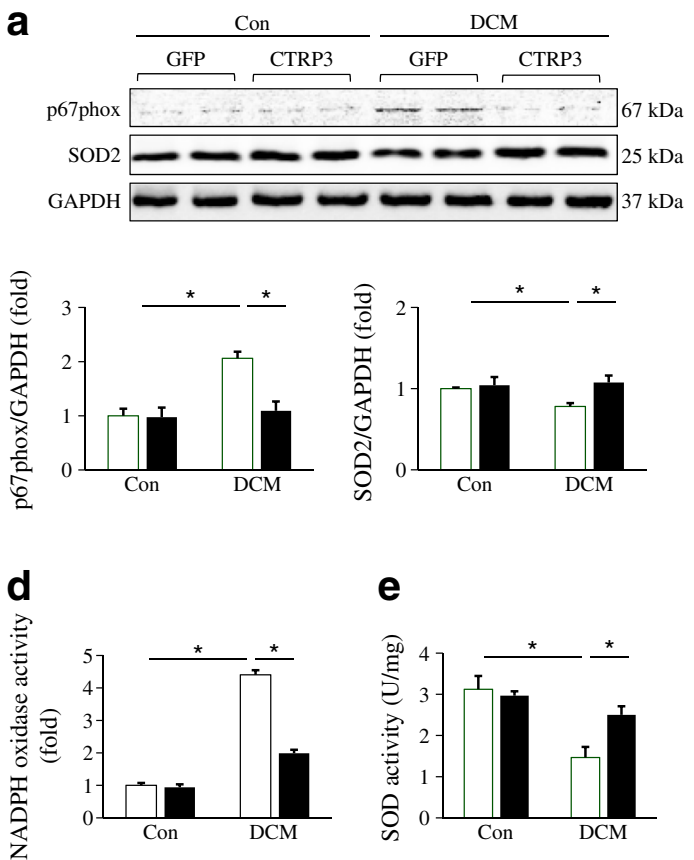

e

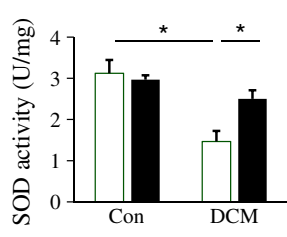

f

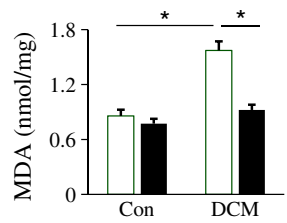

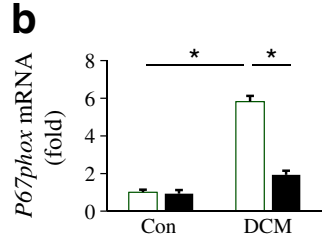

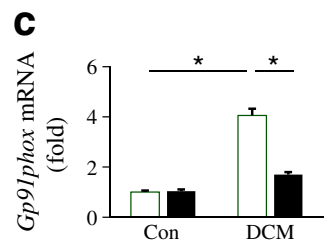

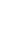

g

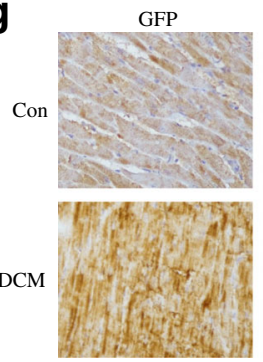

CTRP3
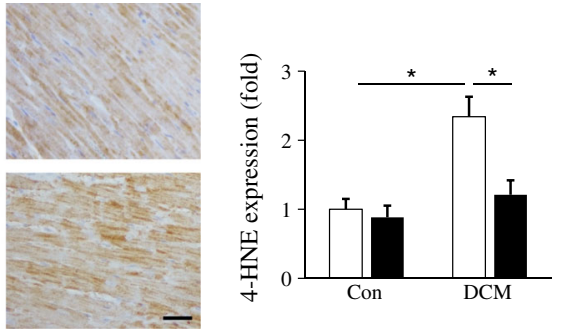

CoA carboxylase (ESM Fig. 3). Intriguingly, administration of CTRP3 induced a substantial reduction of ROS in H9c2 cells exposed to $\mathrm{HG}$ for $24 \mathrm{~h}$ (Fig. $7 \mathrm{~b}-\mathrm{d}$ ). Considering the activation of AMPK $\alpha$ and Akt after CTRP3 exposure, we further assessed whether AMPK $\alpha$ or Akt was involved in the CTRP3-mediated protection. H9c2 cells were infected with adenovirus to knock down AMPK $\alpha 2$ or were treated with an Akt inhibitor. Surprisingly, AMPK $\alpha 2$ knockdown but not the inhibition of Akt blocked the protection afforded by CTRP3 against ROS production (Fig. 7b-d). Fluorescence microscopic observations of DCFH-DA-labelled cells and detection of p67phox confirmed that CTRP3 attenuated HGinduced ROS by activating AMPK $\alpha$ (Fig. 7b, e). Given the inhibitory role of CTRP3 on inflammation in vivo, we then assessed whether CTRP3 could affect HG-induced inflammation in vitro. H9c2 cells treated with CTRP3 ( $3 \mu \mathrm{g} / \mathrm{ml}$ for $24 \mathrm{~h}$ ) had lower mRNA levels of Tnf- $\alpha$ at baseline when compared with untreated cells (Fig. 7f, g). Moreover, CTRP3 attenuated HG-induced inflammation in H9c2 cells, as shown by the altered mRNA levels of Tnf- $\alpha$ and Mcp-1 (Fig. 7f-i). The changes in these levels were blunted by AMPK $\alpha 2$ deficiency and were unaffected by Akt inhibition (Fig. $7 \mathrm{f}-\mathrm{i}$ ).
CTRP3 inhibited HG-induced cell death by activating AMPK $\alpha$ in H9c2 cells Next, we determined whether CTRP3 protected against HG-induced death of myocytes. $\mathrm{H} 9 \mathrm{c} 2$ cells exposed to $\mathrm{HG}$ for $72 \mathrm{~h}$ had decreased cell viability and after CTRP3 treatment the cell viability was increased (Fig. 8a, b). AMPK $\alpha 2$ knockdown but not the inhibition of Akt abolished the protection of CTRP3 against HG-induced cell death (Fig. 8b, c). HG also downregulated BCL-2 and upregulated BAX in H9c2 cells (ESM Fig. 4); however, these alterations were blocked by CTRP3 treatment. The effects of CTRP3 were abolished by AMPK $\alpha 2$ deficiency (ESM Fig. 4a). Given the increased TNF- $\alpha$ during the process of DCM, the H9c2 cells were treated with TNF- $\alpha(5 \mathrm{ng} / \mathrm{ml})$. CTRP3 significantly inhibited TNF- $\alpha$-induced apoptosis, an effect which was reversed by AMPK $\alpha 2$ deficiency but not by Akt inhibition (Fig. 8d, e). Confirmation was provided by western blot analysis of BCL-2 and BAX (ESM Fig. 4b). $\mathrm{H}_{2} \mathrm{O}_{2}$ was used to induce myocyte apoptosis. AMPK $\alpha 2$ deficiency completely blocked the protection provided by CTRP3 against $\mathrm{H}_{2} \mathrm{O}_{2}$-induced apoptosis (Fig. 8f). This was further confirmed by TUNEL staining (ESM Fig. 4c). 
Fig. 5 CTRP3 attenuated diabetes-induced inflammation. (a-d) PCR analysis of myocardial Tnf- $\alpha, I l-1 \beta, I l-6$ and $M c p-1$ mRNA in rats with diabetes $(n=6)$. (e) Immunohistochemical analysis of TNF- $\alpha$ in diabetic hearts. Representative images and quantification $(n=6)$ are shown. Scale bar, $300 \mu \mathrm{m}$. (f) Western blot analysis of TNF- $\alpha$ in diabetic hearts $(n=6)$. (g) TUNEL staining in diabetic hearts $(n=6)$. Arrows indicate apoptotic cells. (h) Western blot analysis of BCL2 and BAX in diabetic hearts $(n=6)$. Scale bar, $10 \mu \mathrm{m}$. White bars, GFP; black bars, CTRP3. ${ }^{*} p<0.05$ for indicated comparisons. Con, control (no diabetes)
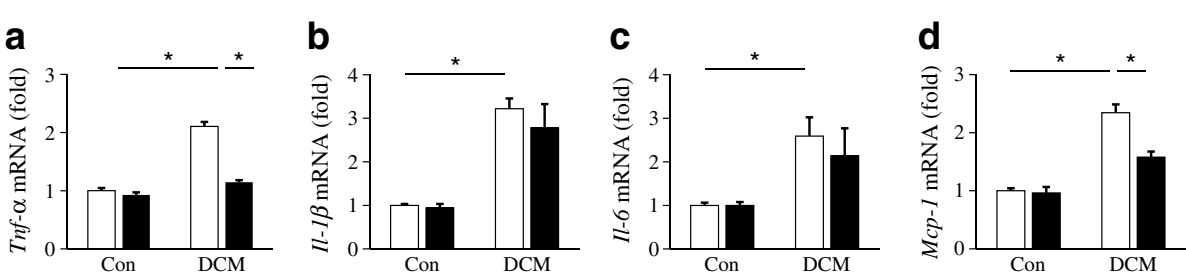

e
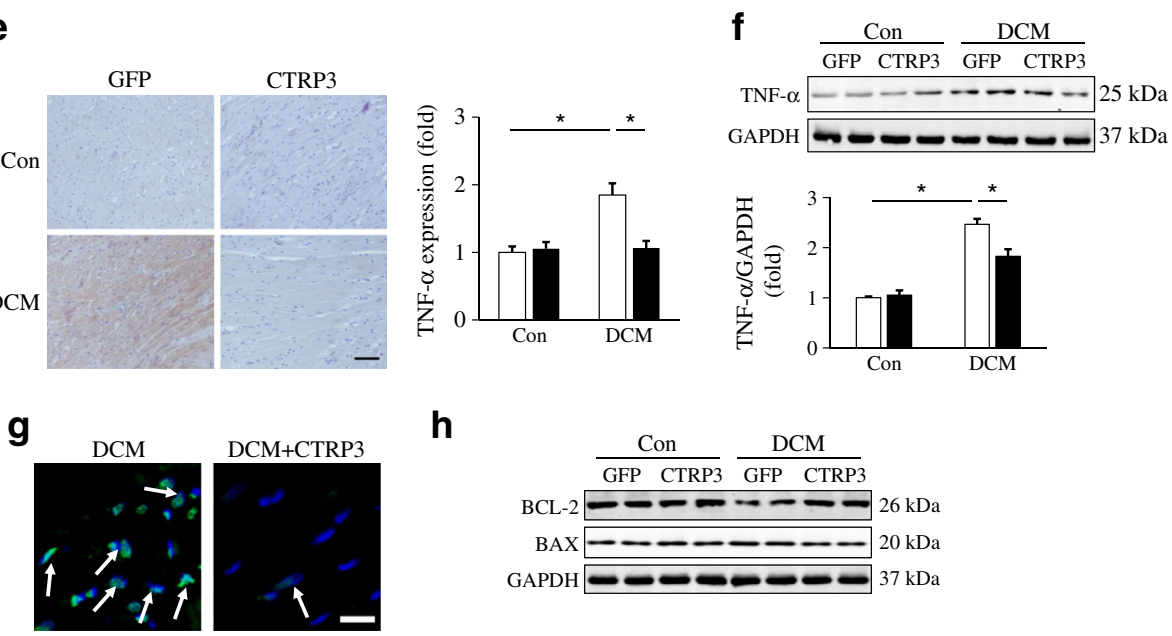

h
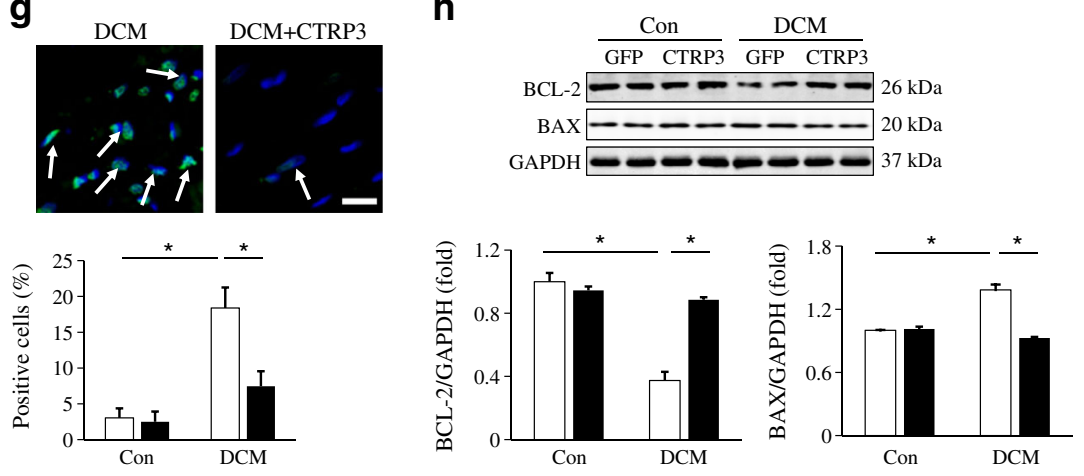

\begin{abstract}
AMPK $\alpha$ deficiency counteracted the protective effects of CTRP3 in vivo Subsequently, we investigated whether the morphological changes observed in rats after overexpression of CTRP3 were reversed by AMPK $\alpha$ deficiency. Intramyocardial injection of Ad-shAmpk $\alpha$ resulted in the downregulation of AMPK $\alpha$ in the hearts (Fig. 9a). Rats in the shAmpk $\alpha+C T R P 3+D C M$ group exhibited an aggravated phenotype, as indicated by decreased heart weight in relation to tibia length, restricted LVIDd, reduced FS and augmented haemodynamic alteration (Fig. 9b-f).
\end{abstract}

The precise mechanism by which CTRP3 activates AMPK $\alpha$ A previous study demonstrated that the receptor of CTRP3 may be a G protein-coupled receptor (GPCR) and that CTRP3 increased the level of cAMP in TM3 Leydig cells [26]. We found that CTRP3 increased cAMP in H9c2 cells (Fig. 10a). cAMP is produced by adenylate cyclase; therefore, an adenylate cyclase inhibitor ( $2^{\prime} 5^{\prime}$-dd-Ado) was used to investigate the possible role. The inhibitor $(200 \mu \mathrm{mol} / 1,2 \mathrm{~h})$ blocked CTRP3-induced AMPK $\alpha$ activation (Fig. 10b). Next, a PKA inhibitor and small interfering RNA of EPAC
Fig. 6 CTRP3 activated AMPK $\alpha$ and Akt in the diabetic hearts $(n=6)$. White bars, control+GFP; light grey bars, control+CTRP3; black bars, $\mathrm{DCM}+\mathrm{GFP}$; dark grey bars, $\mathrm{DCM}+\mathrm{CTRP} 3 . * p<0.05$ for indicated comparisons. ACC, acetyl-CoA carboxylase; NRF2, nuclear factor-E2 p45-related factor 2; p-, phosphorylated; T-, total. Con, control (no diabetes)
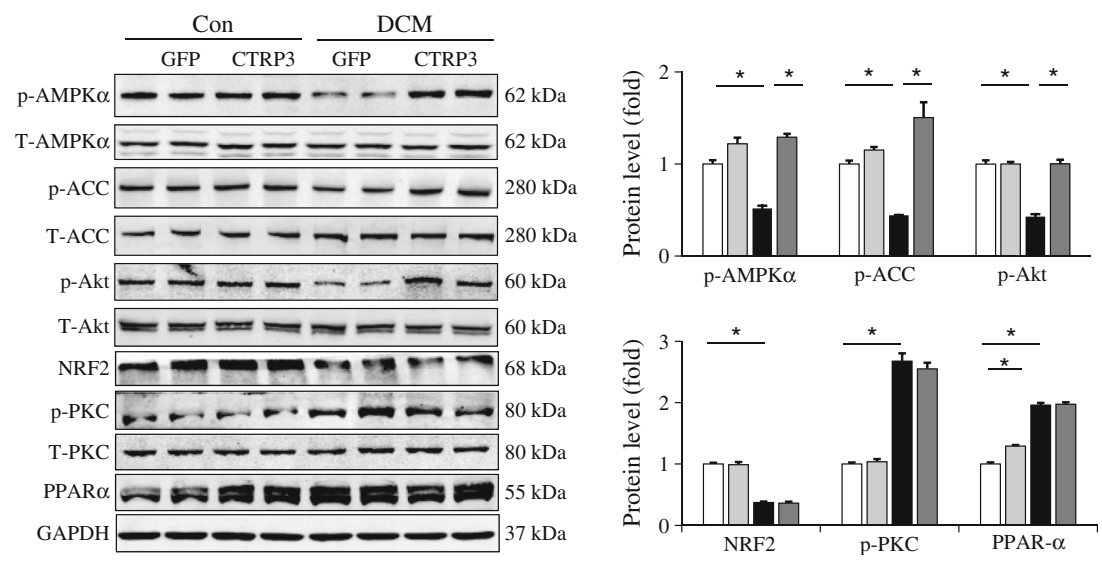


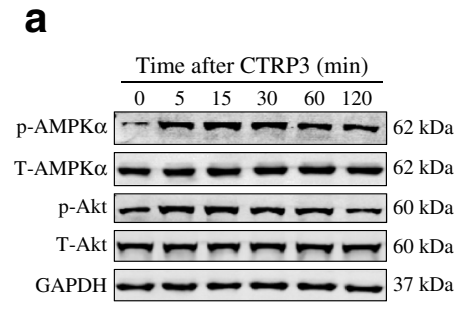

C

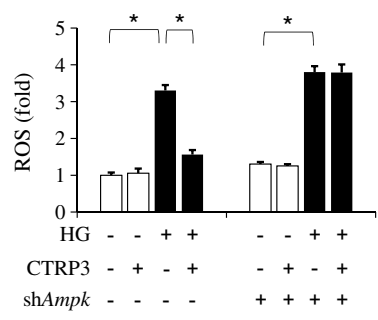

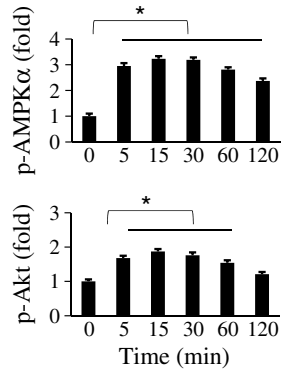

d

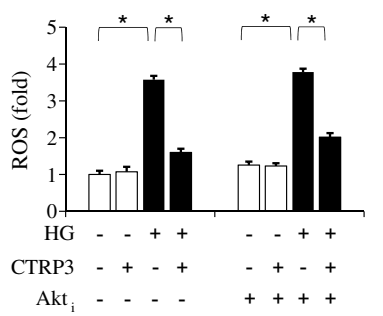

b

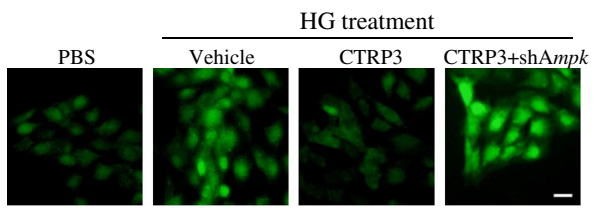

e

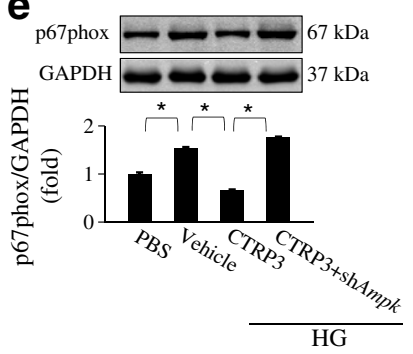

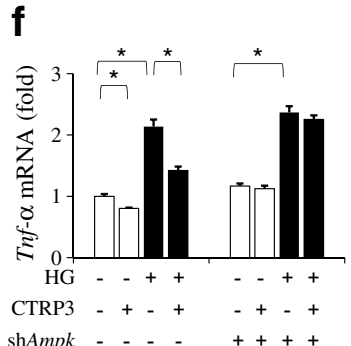

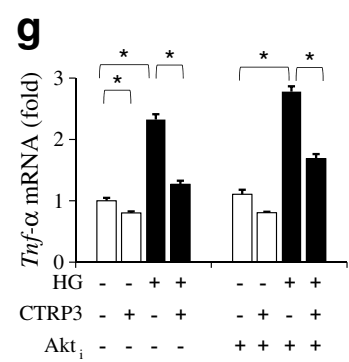

Fig. 7 CTRP3 suppressed HG-induced production of ROS and inflammation in $\mathrm{H} 9 \mathrm{c} 2$ cells. (a) CTRP3 $(3 \mu \mathrm{g} / \mathrm{ml})$ activated AMPK $\alpha$ and Akt $(n=4)$. (b) Representative fluorescence microscopy images of DCF-DA fluorescence. Scale bar, $100 \mu \mathrm{m}$. (c) AMPK $\alpha$ deficiency offset the protection afforded by CTRP3 against ROS induced by HG $(24 \mathrm{~h})(n=6)$. (d)

(siEpac) were used. Knockdown of EPAC but not inhibition of PKA prevented CTRP3-induced AMPK $\alpha$ activation (Fig. 10b and ESM Fig. 5). Activation of AMPK $\alpha$ was inhibited by the MEK inhibitor U0126 (Fig. 10b). Then, we examined how MEK activation is related to the activation of AMPK $\alpha$. However, a CaMKK II inhibitor (STO-609) had no effect on the activation of AMPK $\alpha$ (Fig. 10b). CTRP3 activated liver kinase B1 (LKB1) and this activation was prevented by adenylate cyclase inhibition, EPAC deficiency or MEK inhibition (Fig. 10b). Moreover, the protection provided by CTRP3 against HG-induced cell loss was also abolished by 2'5'-dd-Ado, EPAC deficiency and U0126 (Fig. 10c).

\section{Discussion}

To our knowledge, this is the first report to demonstrate that knockdown of CTRP3 in cardiomyocytes results in oxidative stress, inflammation and cell death and that overexpression of CTRP3 in the heart protects against diabetes-related cardiac
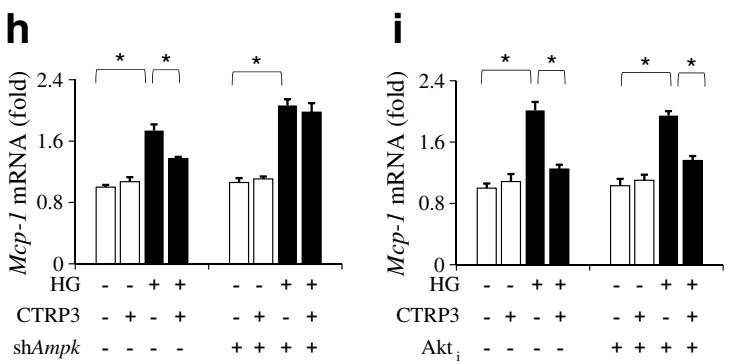

Akt inhibition $\left(\mathrm{Akt}_{\mathrm{i}}\right)$ did not affect the production of ROS $(n=6)$. (e) Protein level of P67phox in H9c2 cells $(n=5)$. ( $(\mathbf{f}-\mathbf{i})$ AMPK $\alpha$ deficiency but not $\mathrm{Akt}_{\mathrm{i}}$ offset the protection afforded by CTRP3 against inflammation induced by HG in $\mathrm{H} 9 \mathrm{c} 2$ cells $(24 \mathrm{~h})(n=6) . * p<0.05$ for indicated comparisons. p-, phosphorylated; T-, total

dysfunction, oxidative damage, inflammation and cell death in vivo. In this study, CTRP3 also attenuated HG-induced production of ROS and inflammation, and reduced HG-induced cell loss in vitro. Moreover, we found that the protective effect of CTRP3 was mediated by activation of AMPK $\alpha$ and that AMPK deficiency could offset the protective effect of CTRP3 in vivo. For the first time, we found that CTRP3 activated AMPK $\alpha$ via the cAMP-EPAC-MEKLKB1 pathways.

Previous studies have noted the association between CTRP3 and metabolic and cardiovascular diseases. Circulating CTRP3 was significantly decreased in mice fed a high-fat diet for 12 weeks [16]. Li et al also found that the levels of CTRP 3 were decreased in epididymal adipose tissue of rats in a model of type 2 diabetes [27]. Furthermore, a crosssectional study revealed that decreased CTRP3 levels were closely associated with obesity in humans [28]. Consistent with these findings, we also showed that CTRP3 levels in diabetic rat hearts were significantly decreased. CTRP3 deficiency at baseline can result in oxidative stress, inflammation and cell death, recapitulating the phenotype of DCM in vitro. 

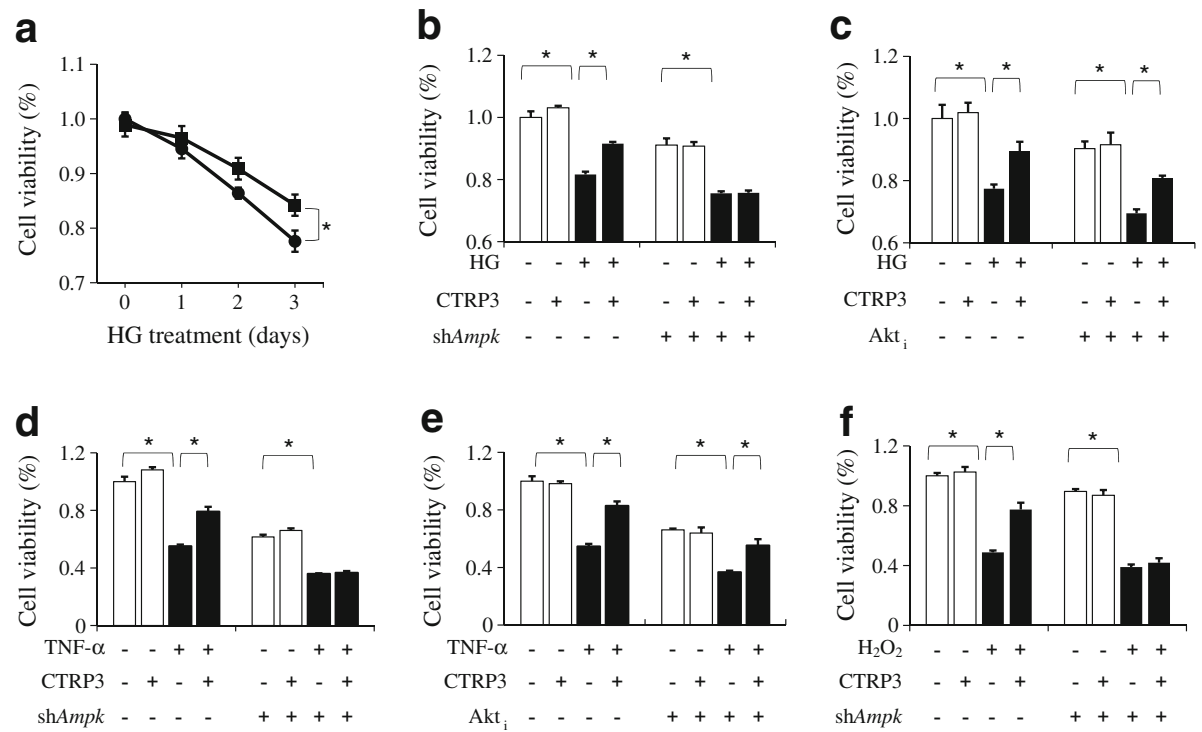

Fig. 8 CTRP3 blocked HG-induced cell death in H9c2 cells. (a) CTRP3 increased cell viability of $\mathrm{H} 9 \mathrm{c} 2$ cells $(n=6)$. Circles, PBS; squares, CTRP3. (b) AMPK $\alpha$ deficiency offset the protection afforded by CTRP3 against HG (72 h)-induced death of H9c2 cells $(n=6)$. (c) The Akt1/2 kinase inhibitor $\left(\mathrm{Akt}_{\mathrm{i}}\right)(1 \mu \mathrm{mol} / \mathrm{l}$ for $72 \mathrm{~h})$ had no effect on death of H9c2 cells exposed to HG for $72 \mathrm{~h}(n=6)$. (d) AMPK $\alpha$ deficiency offset

This observation prompted us to investigate whether optimising CTRP3 levels in diabetic hearts could attenuate diabetes-related cardiac injury. As expected, overexpression of CTRP3 in the hearts or systemic administration of CTRP3 could alleviate diabetes-related cardiac dysfunction,
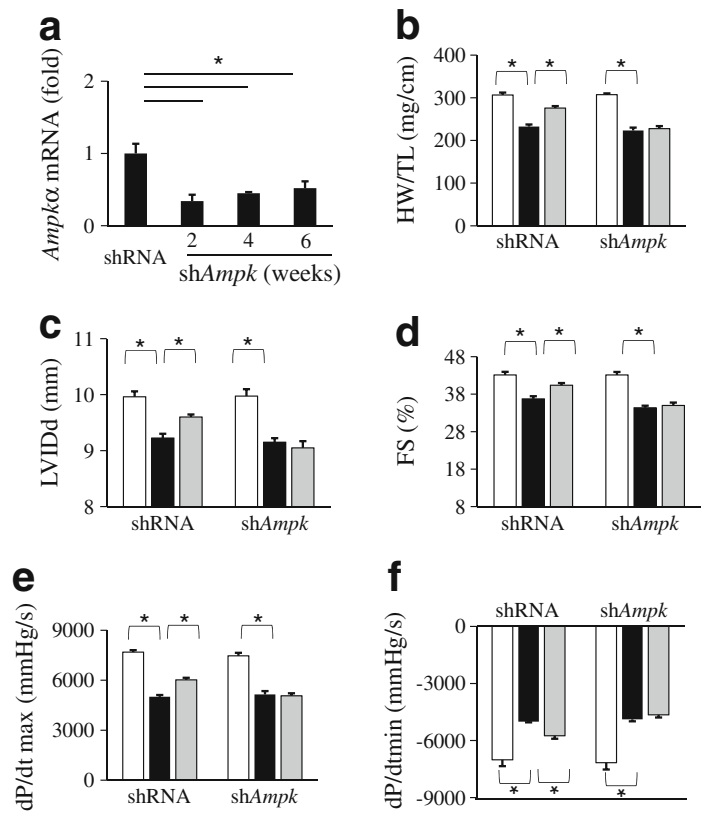

Fig. 9 AMPK $\alpha$ deficiency offset the protective effects of CTRP3 in vivo. (a) mRNA level of Ampk $\alpha(n=6)$. (b) Ratio of heart weight (HW) to tibia length (TL) $(n=10)$. (c, d) Alteration of FS (c) and LVIDd (d) $(n=8)$. (e, f) Alteration of haemodynamic measurements $(n=8)$. For $(\mathbf{b}-\mathbf{f})$ : white bars, control; black bars, DCM+GFP; grey bars, DCM+CTRP3. $* p<0.05$ for indicated comparisons the protection of CTRP 3 against death of $\mathrm{H} 9 \mathrm{c} 2$ cells caused by TNF- $\alpha$ $(5 \mathrm{ng} / \mathrm{ml}$ for $24 \mathrm{~h})(n=6)$. (e) $\mathrm{Akt}_{\mathrm{i}}(1 \mu \mathrm{mol} / \mathrm{l}$ for $72 \mathrm{~h})$ had no effect on death of H9c2 cells caused by TNF- $\alpha(5 \mathrm{ng} / \mathrm{ml}$ for $24 \mathrm{~h})(n=6)$. (f) AMPK $\alpha$ deficiency offset the protection afforded by CTRP 3 against death of $\mathrm{H} 9 \mathrm{c} 2$ cells induced by $\mathrm{H}_{2} \mathrm{O}_{2}(200 \mu \mathrm{mol} / 1$ for $12 \mathrm{~h})(n=6)$. $* p<0.05$ for indicated comparisons

indicating that CTRP3 is a promising target for the treatment of DCM. Intriguingly, overexpression of CTRP3 in normal hearts did not lead to any abnormalities, indicating that gene-based therapy targeting CTRP3 is a potential strategy.

Accumulating evidence demonstrates that an exponential increase in oxidative stress promotes the process of DCM [29, 30]. An increase in ROS, observed in myocytes treated with HG [31] and in cardiomyocytes isolated from diabetic mice $[29,30]$, caused lipid peroxidation and DNA damage [32, 33], eventually leading to functional abnormalities of the heart. Therefore, it is important to identify suppressors of oxidative stress in diabetic hearts. In contrast to a previous finding that CTRP3 induced ROS in vascular smooth muscle cells [34], here, we provided robust evidence suggesting that CTRP3 protected against diabetes-induced oxidative damage in vivo and blocked HG-induced production of ROS in vitro. This was confirmed by our finding that knockdown of CTRP3 increased ROS levels in $\mathrm{H} 9 \mathrm{c} 2$ cells. Inflammation is another characteristic of DCM. Diabetic hearts display increased levels of TNF- $\alpha$ and IL-1 $\beta$ [35], which can result in cardiac dysfunction [36] and affect cardiac contractility [37]. CTRP3 has been demonstrated to suppress inflammation. Kopp et al found that CTRP3 served as an endogenous antagonist of lipopolysaccharide (LPS) and blocked the production of TNF- $\alpha$, IL-6 and MCP-1 [19]. Furthermore, CTRP3 suppressed systemic inflammation in mice treated with LPS [19]. The inhibitory effects of CTRP3 were further confirmed by studies using CTRP 3 transgenic animals, showing that mice overexpressing CTRP3 had lower circulating levels of TNF- $\alpha$ after high-fat feeding compared with wild-type 
Fig. 10 The precise mechanism by which CTRP 3 activates AMPK $\alpha$. (a) cAMP level in $\mathrm{H} 9 \mathrm{c} 2$ cells treated with CTRP3 for $2 \mathrm{~h}$ $(n=6)$. (b) p-LKB1 and pAMPK $\alpha$ levels in $\mathrm{H} 9 \mathrm{c} 2$ cells treated with 2'5'-dd-Ado (a specific adenylate cyclase inhibitor, $200 \mu \mathrm{mol} / \mathrm{l}$ ) or U0126 (a MEK inhibitor, $10 \mu \mathrm{mol} / \mathrm{l}$ ) for $2 \mathrm{~h}$. (c) Cell viability of $\mathrm{H} 9 \mathrm{c} 2$ cells $(n=6) . * p<0.05$ for indicated comparisons. p-, phosphorylated; T-, total

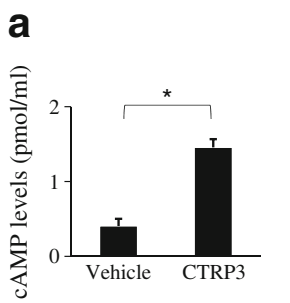

b

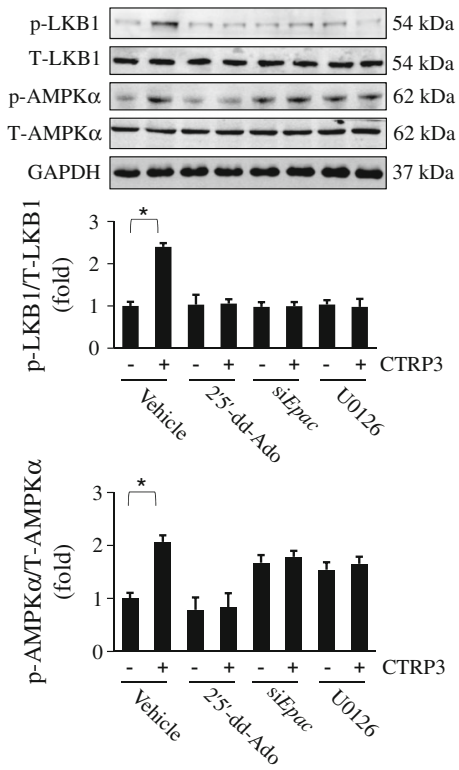

C

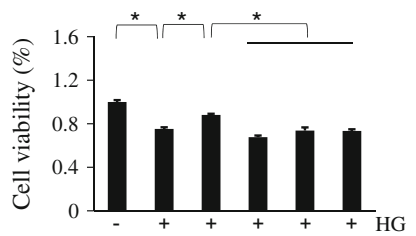

+ CTRP

2'5'-dd-Ado

- siEpac

$+\mathrm{U} 0126$ littermate controls [18]. Consistent with these findings, in our study, attenuation of inflammation after CTRP3 treatment was observed in the diabetic rat hearts. We also found that CTRP3 could mitigate HG-induced inflammation in vitro and that knockdown of CTRP3 increased Tnf- $\alpha$ and Mcp-1 levels in vitro. However, Kopp et al reported that CTRP 3 also affected the level of IL-6 [38], which, in our current study, was unaffected by CTRP3.

Cell death is a key feature of STZ-induced cardiomyopathy [39]. Previous studies found that CTRP3 inhibited apoptosis in ischaemic mouse hearts in vivo [15] and suppressed apoptosis of prostate cells in vitro [40]. Consistent with these previous reports, we found that CTRP 3 significantly inhibited diabetes-induced cell death in vivo and HG-induced apoptosis in vitro. In vitro, CTRP3 also blocked cell death induced by TNF- $\alpha$ or $\mathrm{H}_{2} \mathrm{O}_{2}$. Taken together, these observations demonstrate the protective role of CTRP 3 against cell death in diabetic hearts.

Relatively little is known about how CTRP3 exerts its protective effects. CTRP3 is reported to stimulate proliferation via PKC signalling pathways [40] but we failed to observe an alteration in the PKC pathway in diabetic hearts. In contrast to a study showing that CTRP3 had no effect on AMPK $\alpha$ [15], we found that CTRP3 activated Akt and AMPK $\alpha$ in vivo and in vitro, which was consistent with previous findings $[20,41]$. Based on our results, we hypothesise that the effects of CTRP 3 are mediated by Akt or AMPK $\alpha$. Notably, our study indicated that AMPK $\alpha$ deficiency, not inhibition of Akt, abolishes the protection provided by CTRP3 against oxidative stress, inflammation and cell death. This is inconsistent with a previous finding demonstrating that CTRP3 protected mesenchymal stem cells against apoptosis by increasing Akt phosphorylation [42]. We further showed that the inhibitory effects of CTRP3 against diabetes-related cardiac dysfunction were offset by AMPK deficiency in vivo, indicating that the protective effects of CTRP 3 were mediated by AMPK $\alpha$.

A crucial question raised by our data in this study concerns the pathway through which CTRP3 activates AMPK $\alpha$. CTRP3 promotes the production of testosterone via the cAMP-PKA pathway [26]. The data in our study also demonstrated that CTRP3 could increase cAMP, as shown by increased cAMP levels after CTRP 3 administration and the finding that an adenylate cyclase inhibitor blocked CTRP3-induced AMPK $\alpha$ activation. However, the observation that a PKA inhibitor did not affect AMPK $\alpha$ activation caused by CTRP3 indicated that PKA was not involved in the CTRP3-mediated AMPK $\alpha$ activation. cAMP also activated EPAC, which directly activates Rap1 and induces the activation of MEK [43]. We showed that knockdown of EPAC or inhibition of MEK blocked CTRP3mediated AMPK $\alpha$ activation, further indicating the involvement of the cAMP-EPAC-MEK pathway in AMPK $\alpha$ activation induced by CTRP3. Consistent with a previous finding that MEK activated LKB1 [44, 45], a well-known regulator of AMPK $\alpha$, the change in LKB1 in our study correlated directly with AMPK $\alpha$, suggesting a role for LKB1 in the activation of AMPK $\alpha$. Inhibition of CaMKK II had no significant effect on the phosphorylation of AMPK $\alpha$, indicating the dispensable role of CaMKK II in this process. Altogether, our data demonstrated that CTRP3 activated AMPK $\alpha$ via the cAMP-EPAC-MEKLKB1 pathways.

In summary, this study identified CTRP3 as a critical negative regulator of DCM via activation of AMPK $\alpha$ and attenuation of oxidative stress, inflammation and cell death. Our study provides evidence that CTRP 3 could be a promising therapeutic approach for the treatment of DCM. 
Data availability The data that support the findings of this study are available from the corresponding author upon reasonable request.

Funding This work was supported by grants from the National Natural Science Foundation of China (no. 81270303, 81470516, 81470402) and the Key Project of the National Natural Science Foundation (no. 81530012).

Duality of interest The authors declare that there is no duality of interest associated with this manuscript.

Contribution statement Z-GM, Y-PY, S-CX, W-YW, C-RX and QQW performed the study, analysed and interpreted the data and wrote the manuscript. Z-GM, Y-PY, S-CX, W-YW and XZ contributed to acquisition of data and manuscript preparation and revision. Z-GM and Q-ZT conceived the hypothesis, participated in the experimental design, data interpretation and manuscript preparation and revision. H-HL and JN contributed to data collection and interpretation of data and manuscript drafting. All authors approved the final version of the manuscript. Q-ZT is the guarantor of this work.

\section{References}

1. Boudina S, Abel ED (2007) Diabetic cardiomyopathy revisited. Circulation 115:3213-3223

2. Bugger H, Abel ED (2014) Molecular mechanisms of diabetic cardiomyopathy. Diabetologia 57:660-671

3. Kannel WB, Hjortland M, Castelli WP (1974) Role of diabetes in congestive heart failure: the Framingham study. Am J Cardiol 34: 29-34

4. Devereux RB, Roman MJ, Paranicas M et al (2000) Impact of diabetes on cardiac structure and function: the strong heart study. Circulation 101:2271-2276

5. Kannel WB, McGee DL (1979) Diabetes and cardiovascular disease. The Framingham study. JAMA 241:2035-2038

6. Redfield MM, Jacobsen SJ, Burnett JJ, Mahoney DW, Bailey KR, Rodeheffer RJ (2003) Burden of systolic and diastolic ventricular dysfunction in the community: appreciating the scope of the heart failure epidemic. JAMA 289:194-202

7. Hayat SA, Patel B, Khattar RS, Malik RA (2004) Diabetic cardiomyopathy: mechanisms, diagnosis and treatment. Clin Sci (Lond) 107:539-557

8. Cai L, Kang YJ (2001) Oxidative stress and diabetic cardiomyopathy: a brief review. Cardiovasc Toxicol 1:181-193

9. Cai L, Kang YJ (2003) Cell death and diabetic cardiomyopathy. Cardiovasc Toxicol 3:219-228

10. Westermann D, Van Linthout S, Dhayat $S$ et al (2007) Tumor necrosis factor-alpha antagonism protects from myocardial inflammation and fibrosis in experimental diabetic cardiomyopathy. Basic Res Cardiol 102:500-507

11. Inoguchi T, Li P, Umeda F et al (2000) High glucose level and free fatty acid stimulate reactive oxygen species production through protein kinase $\mathrm{C}$-dependent activation of $\mathrm{NAD}(\mathrm{P}) \mathrm{H}$ oxidase in cultured vascular cells. Diabetes 49:1939-1945

12. Barouch LA, Berkowitz DE, Harrison RW, O'Donnell CP, Hare JM (2003) Disruption of leptin signaling contributes to cardiac hypertrophy independently of body weight in mice. Circulation 108:754 759

13. Fubini B, Hubbard A (2003) Reactive oxygen species (ROS) and reactive nitrogen species (RNS) generation by silica in inflammation and fibrosis. Free Radic Biol Med 34:1507-1516

14. Schaffler A, Buechler C (2012) CTRP family: linking immunity to metabolism. Trends Endocrinol Metab 23:194-204
15. Yi W, Sun Y, Yuan Y et al (2012) C1q/tumor necrosis factor-related protein-3, a newly identified adipokine, is a novel antiapoptotic, proangiogenic, and cardioprotective molecule in the ischemic mouse heart. Circulation 125:3159-3169

16. Peterson JM, Wei Z, Wong GW (2010) C1q/TNF-related protein-3 (CTRP3), a novel adipokine that regulates hepatic glucose output. J Biol Chem 285:39691-39701

17. Peterson JM, Seldin MM, Wei Z, Aja S, Wong GW (2013) CTRP3 attenuates diet-induced hepatic steatosis by regulating triglyceride metabolism. Am J Physiol Gastrointest Liver Physiol 305:G214 G224

18. Petersen PS, Wolf RM, Lei X, Peterson JM, Wong GW (2016) Immunomodulatory roles of CTRP3 in endotoxemia and metabolic stress. Physiol Rep 4, e12735

19. Schmid A, Kopp A, Hanses F, Karrasch T, Schaffler A (2014) C1q/ TNF-related protein-3 (CTRP-3) attenuates lipopolysaccharide (LPS)-induced systemic inflammation and adipose tissue Erk-1/-2 phosphorylation in mice in vivo. Biochem Biophys Res Commun 452:8-13

20. Wu D, Lei H, Wang JY et al (2015) CTRP3 attenuates post-infarct cardiac fibrosis by targeting Smad3 activation and inhibiting myofibroblast differentiation. J Mol Med (Berl) 93:1311-1325

21. Ma ZG, Dai J, Zhang WB et al (2016) Protection against cardiac hypertrophy by geniposide involves the GLP-1 receptor/AMPK $\alpha$ signalling pathway. Br J Pharmacol 173:1502-1516

22. Ma Z, Dai J, Wei W et al (2016) Asiatic acid protects against cardiac hypertrophy through activating $\mathrm{AMPK} \alpha$ signalling pathway. Int J Biol Sci 12:861-871

23. Wei WY, Ma ZG, Xu SC, Zhang N, Tang QZ (2016) Pioglitazone Protected against Cardiac Hypertrophy via Inhibiting AKT/GSK3 $\beta$ and MAPK Signaling Pathways. PPAR Res 2016:9174190

24. Simpson P, McGrath A, Savion S (1982) Myocyte hypertrophy in neonatal rat heart cultures and its regulation by serum and by catecholamines. Circ Res 51:787-801

25. Su H, Yuan Y, Wang XM et al (2013) Inhibition of CTRP9, a novel and cardiac-abundantly expressed cell survival molecule, by $\mathrm{TNF} \alpha$-initiated oxidative signaling contributes to exacerbated cardiac injury in diabetic mice. Basic Res Cardiol 108:315

26. Otani M, Kogo M, Furukawa S, Wakisaka S, Maeda T (2012) The adiponectin paralog C1q/TNF-related protein 3 (CTRP3) stimulates testosterone production through the $\mathrm{cAMP} / \mathrm{PKA}$ signaling pathway. Cytokine 58:238-244

27. Li X, Jiang L, Yang M, Wu YW, Sun SX, Sun JZ (2014) Expression of CTRP3, a novel adipokine, in rats at different pathogenic stages of type 2 diabetes mellitus and the impacts of GLP-1 receptor agonist on it. J Diabetes Res 2014:398518

28. Wolf RM, Steele KE, Peterson LA, Magnuson TH, Schweitzer MA, Wong GW (2015) Lower circulating C1q/TNF-related protein-3 (CTRP3) levels are associated with obesity: a cross-sectional study. PLoS ONE 10, e133955

29. Cai L, Wang Y, Zhou G et al (2006) Attenuation by metallothionein of early cardiac cell death via suppression of mitochondrial oxidative stress results in a prevention of diabetic cardiomyopathy. J Am Coll Cardiol 48:1688-1697

30. Kajstura J, Fiordaliso F, Andreoli AM et al (2001) IGF-1 overexpression inhibits the development of diabetic cardiomyopathy and angiotensin II-mediated oxidative stress. Diabetes 50:1414-1424

31. Cai L, Li W, Wang G, Guo L, Jiang Y, Kang YJ (2002) Hyperglycemia-induced apoptosis in mouse myocardium: mitochondrial cytochrome C-mediated caspase-3 activation pathway. Diabetes 51:1938-1948

32. Dandona P, Thusu K, Cook S et al (1996) Oxidative damage to DNA in diabetes mellitus. Lancet 347:444-445

33. Hinokio Y, Suzuki S, Hirai M, Chiba M, Hirai A, Toyota T (1999) Oxidative DNA damage in diabetes mellitus: its association with diabetic complications. Diabetologia 42:995-998 
34. Feng H, Wang JY, Zheng M et al (2016) CTRP3 promotes energy production by inducing mitochondrial ROS and up-expression of PGC-1alpha in vascular smooth muscle cells. Exp Cell Res 341: 177-186

35. Westermann D, Rutschow S, Jager S et al (2007) Contributions of inflammation and cardiac matrix metalloproteinase activity to cardiac failure in diabetic cardiomyopathy: the role of angiotensin type 1 receptor antagonism. Diabetes 56:641-646

36. Bryant D, Becker L, Richardson J et al (1998) Cardiac failure in transgenic mice with myocardial expression of tumor necrosis factor-alpha. Circulation 97:1375-1381

37. Yokoyama T, Vaca L, Rossen RD, Durante W, Hazarika P, Mann DL (1993) Cellular basis for the negative inotropic effects of tumor necrosis factor-alpha in the adult mammalian heart. J Clin Invest 92: 2303-2312

38. Kopp A, Bala M, Buechler C et al (2010) C1q/TNF-related protein3 represents a novel and endogenous lipopolysaccharide antagonist of the adipose tissue. Endocrinology 151:5267-5278

39. Fiordaliso F, Li B, Latini R et al (2000) Myocyte death in streptozotocin-induced diabetes in rats in angiotensin II- dependent. Lab Investig 80:513-527
40. Hou Q, Lin J, Huang W, Li M, Feng J, Mao X (2015) CTRP3 Stimulates proliferation and anti-apoptosis of prostate cells through PKC signaling pathways. Plos One 10, e134006

41. Kim JY, Min JY, Baek JM et al (2015) CTRP3 acts as a negative regulator of osteoclastogenesis through AMPK-c-Fos-NFATc1 signaling in vitro and RANKL-induced calvarial bone destruction in vivo. Bone 79:242-251

42. Hou M, Liu J, Liu F, Liu K, Yu B (2014) C1q tumor necrosis factorrelated protein-3 protects mesenchymal stem cells against hypoxiaand serum deprivation-induced apoptosis through the phosphoinositide 3-kinase/Akt pathway. Int J Mol Med 33:97-104

43. de Rooij J, Zwartkruis FJ, Verheijen MH et al (1998) Epac is a Rap1 guanine-nucleotide-exchange factor directly activated by cyclic AMP. Nature 396:474-477

44. Sapkota GP, Kieloch A, Lizcano JM et al (2001) Phosphorylation of the protein kinase mutated in Peutz-Jeghers cancer syndrome, LKB1/STK11, at Ser431 by p90(RSK) and cAMP-dependent protein kinase, but not its farnesylation at Cys(433), is essential for LKB1 to suppress cell vrowth. J Biol Chem 276:19469-19482

45. Fu D, Wakabayashi Y, Lippincott-Schwartz J, Arias IM (2011) Bile acid stimulates hepatocyte polarization through a cAMP-EpacMEK-LKB1-AMPK pathway. Proc Natl Acad Sci U S A 108: $1403-1408$ 